\title{
Corrigendum: Artesunate Suppresses Choroidal Melanoma Vasculogenic Mimicry Formation and Angiogenesis via the Wnt/CaMKII Signaling Axis
}

\author{
Bochao Geng ${ }^{1}$, Yuanzhang Zhu ${ }^{1}$, Yingying Yuan ${ }^{2}$, Jingyi Bai ${ }^{1}$, Zhizhi Dou ${ }^{1}$, \\ Aihua Sui ${ }^{3}$ and Wenjuan Luo ${ }^{1 *}$ \\ ${ }^{1}$ Department of Ophthalmology, The Affiliated Hospital of Qingdao University, Qingdao, China, ${ }^{2}$ Department of Physiology \\ and Pathophysiology, School of Basic Medicine, Qingdao University, Qingdao, China, ${ }^{3}$ Central Laboratory, The Affiliated \\ Hospital of Qingdao University, Qingdao, China
}

\section{OPEN ACCESS}

Edited and reviewed by: Jill Kolesar,

University of Kentucky, United States

*Correspondence:

Wenjuan LuO

luowenjuan@qduhospital.cn

Specialty section:

This article was submitted to Pharmacology of Anti-Cancer Drugs,

a section of the journal

Frontiers in Oncology

Received: 07 February 2022 Accepted: 14 February 2022

Published: 01 March 2022

Citation:

Geng B, Zhu Y, Yuan Y, Bai J, Dou Z, Sui $A$ and Luo W (2022) Corrigendum: Artesunate Suppresses Choroidal Melanoma Vasculogenic Mimicry

Formation and Angiogenesis via the Wnt/CaMKII Signaling Axis.

Front. Oncol. 12:870805. doi: 10.3389/fonc.2022.870805
Keywords: artesunate, choroidal melanoma, vasculogenesis mimicry, angiogenesis, VE-cadherin

\section{A Corrigendum on:}

Artesunate Suppresses Choroidal Melanoma Vasculogenic Mimicry Formation and Angiogenesis via the Wnt/CaMKII Signaling Axis

By Geng B, Zhu Y, Yuan Y, Bai J, Dou Z, Sui A and Luo W (2021). Front. Oncol. 11:714646. doi: $10.3389 /$ fonc.2021.714646.

In the original article, there was a mistake in Figure 7 as published. We recognized by ourselves that the image of the Ctrl siRNA group in OCM-1 cells was misused. The corrected Figure 7 appears below.

The authors apologize for this error and state that this does not change the scientific conclusions of the article in any way. The original article has been updated.

Publisher's Note: All claims expressed in this article are solely those of the authors and do not necessarily represent those of their affiliated organizations, or those of the publisher, the editors and the reviewers. Any product that may be evaluated in this article, or claim that may be made by its manufacturer, is not guaranteed or endorsed by the publisher.

Copyright (C) 2022 Geng, Zhu, Yuan, Bai, Dou, Sui and Luo. This is an open-access article distributed under the terms of the Creative Commons Attribution License (CC BY). The use, distribution or reproduction in other forums is permitted, provided the original author(s) and the copyright owner(s) are credited and that the original publication in this journal is cited, in accordance with accepted academic practice. No use, distribution or reproduction is permitted which does not comply with these terms. 


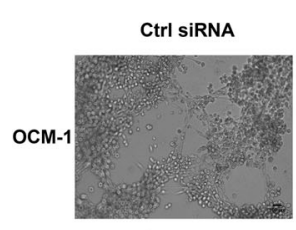

Ctrl siRNA

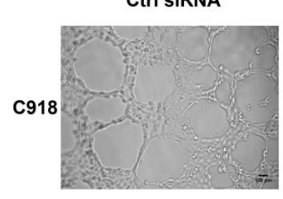

siWnt5a

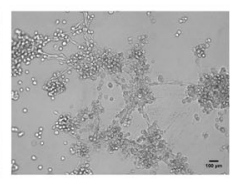

siWnt5a

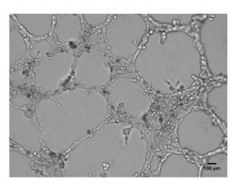

Ctrl siRNA+ART(2.5 $\mu \mathrm{M})$

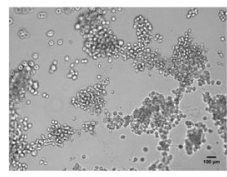

Ctrl SiRNA+ART $(15 \mu \mathrm{M})$

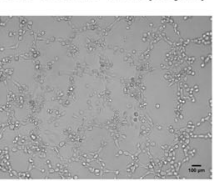

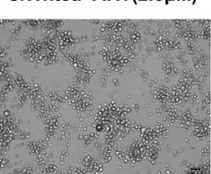

$\operatorname{siWnt5a+ART(15\mu M)}$

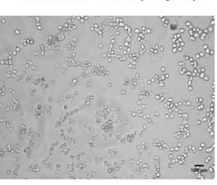

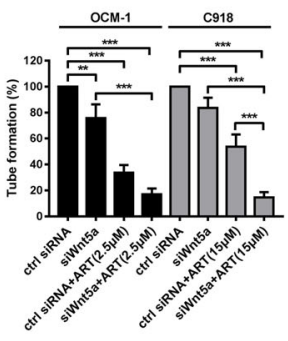

FIGURE 7 | ART interrupted VM formation in CM cells via Wnt5a/CaMKIl pathway. OCM-1 and C918 cells transfected with Wnt5a siRNA or scrambled siRNA subsequently seeded on three-dimensional Matrigel layer culture for $24 \mathrm{~h}$. OCM-1 and C918 cells transfected with Wnt5a siRNA showed a decrease in the number of tubes formed compared with cells transfected with scramble siRNA. Compared with the cells transfected only with small interfering RNA, transfected cells treated with ART have an enhanced inhibitory effect on tube formation. The results are represented as the mean \pm SEM of three independent samples. ${ }^{* *} P<0.01,{ }^{* \star *} P<0.001$. 